\title{
Helicobacter pylori Infection in European Children with Gastro-duodenal Ulcers and Erosions
}

\author{
Patrick Bontems, MD, * Nicolas Kalach, MD, PhD, † Jean Vanderpas, MD, PhD, + Barbara Iwanczak, MD, $\S$ \\ Thomas Casswall, MD, PhD, I Sibylle Koletzko, MD, PhD,ll Giuseppina Oderda, MD, PhD, ** \\ Maria José Martinez-Gomez, MD, †† Pedro Urruzuno, MD, $+\neq$ Angelika Kindermann, MD, $\S \S$ \\ Josef Sykora, MD, 9 I Gabor Veres, MD, PhD, III Eleftheria Roma-Giannikou, MD, PhD, *** \\ Ender Pehlivanoglu, MD, PhD, †† Francis Megraud, MD, PhD, + tt and Samy Cadranel, MD*
}

\begin{abstract}
Background: To analyze risk factors associated with gastro-duodenal ulcers and erosions in children.

Methods: Open, prospective, multicenter, case-control study carried out in 11 European countries in patients with gastric or duodenal ulcers/erosions and 2 age-matched controls each. Possible risk factors were recorded. Logistic regression models were performed with adjustment for centers and age groups.
\end{abstract}

Results: Seven-hundred thirty-two patients (244 cases, 153 with erosions only and 91 with ulcers, and 488 controls) were recruited. Children receiving antimicrobials or acid suppressive drugs before endoscopy were excluded (202 cases/390 controls remained for risk factor analysis). Helicobacter pylori was detected more frequently in cases than controls but only in $32.0 \%$ versus $20.1 \%$ in controls $(P=0.001)$. Independent exposure factors for gastric ulcers were male gender $(P=0.001)$, chronic neurologic disease $(P=0.015)$, chronic renal disease $(P<0.001)$ and nonsteroidal anti-inflammatory drug consumption $(P=0.035)$. Exposure factors for duodenal ulcers were H. pylori infection $(P<0.001)$ and steroid consumption $(P=0.031)$. Chronic renal disease was the only independent factor associated with gastric erosions $(P=0.026)$, those associated with duodenal erosions being H. pylori infection $(P=0.023)$, active smoking $(P=0.006)$ and chronic arthritis $(P=0.008)$. No risk factor was identified in 97/202 (48.0\%) cases. Conclusions: $H$. pylori remains a risk factor for duodenal, but not for gastric lesions in children in countries with low prevalence of infection. No risk factor could be identified in half of the children with gastro-duodenal ulcers/erosions.

Accepted for publication June 17, 2013.

From the *Paediatric Gastroenterology-Hepatology, Queen Fabiola Children's University Hospital, Université Libre de Bruxelles, Brussels, Belgium; †Saint Vincent de Paul Hospital, Clinic of Paediatrics St Antoine, Groupement des Hôpitaux de l'Institut Catholique de Lille (GH-ICL), Lille, France; $\ddagger$ Medical Microbiology Laboratory, Communicable and Infectious Diseases, Scientific Institute of Public Health, Brussels, Belgium; §Department of Paediatrics, Medical University of Wroclaw, Wroclaw, Poland; ๆDepartment of Paediatrics, Karolinska University Hospital, Stockholm, Sweden; UDr v. Hauner Children's Hospital, University Medical Center, Munich, Germany; **Department of Paediatrics, University of Piemonte Orientale, Novara, Italy; †ंDepartment of Paediatrics, Hospital Universitario Niño Jesús; ††Department of Paediatrics, Hospital 12 de Octobre, Madrid, Spain; $\S \S$ Academic Medical Center, Amsterdam, The Netherlands; $₫$ ฯ Charles University in Prague, Faculty of Medicine in Pilsen, Department of Paediatrics, Prague, Czech Republic; IIIFirst Department of Paediatrics, Semmelweis University, Budapest, Hungary; ***First Department of Paediatrics of Athens University, Athens, Greece; ††Department of Paediatrics, Marmara University Hospital, Istanbul, Turkey; and \$\$Inserm U853, Victor Segalen Bordeaux 2 University, Bordeaux, France.

The authors have no other funding or conflicts of interest to disclose.

Address for correspondence: Patrick Bontems, Pediatric GastroenterologyHepatology, Queen Fabiola Children's University Hospital, Université Libre de Bruxelles, 15 Av JJ Crocq, 1020 Brussels, Belgium. E-mail: patrick.bontems@huderf.be.

Copyright (C) 2013 by Lippincott Williams \& Wilkins

ISSN: 0891-3668/13/3212-1324

DOI: $10.1097 /$ INF.0000000000000005
Key Words: Helicobacter pylori, peptic ulcer disease, gastric and duodenal ulcer, gastric and duodenal erosions, children

(Pediatr Infect Dis J 2013;32:1324-1329)

$H^{2}$ elicobacter pylori is known as an important human pathogen that can cause gastritis and peptic ulcers in adults as well as in children, although the general opinion is that ulcers are rare during childhood and that $H$. pylori related gastro-duodenal lesions occur only decades after acquisition of the infection. ${ }^{1-4}$ However, in a large European study concerning antimicrobial susceptibility of $H$. pylori strains infecting children, gastro-duodenal ulcers were found in $3.5 \%$ of infected dyspeptic children $<6$ years of age, in $4.6 \%$ between the age of 6 and 11 years and in $10.4 \%$ of those $>11$ years. ${ }^{5}$ In another multicenter study evaluating the efficacy of eradication treatments, 64/454 (12.3\%) children presented gastro-duodenal ulcers and/or erosions. ${ }^{6}$ Peptic ulcers also occur in $H$. pylori-negative patients, especially those receiving nonsteroidal anti-inflammatory drugs (NSAIDs), as shown in other studies. ${ }^{7-20}$

In a previous uncontrolled study, ${ }^{1}$ we assessed the frequency of gastro-duodenal ulcers/erosions in dyspeptic children referred for upper gastrointestinal (GI) endoscopy in 14 European countries. Ulcers and/or erosions were observed in 56 of 694 children (8.1\%). Age was identified as an important risk factor, children with ulcers/ erosions being significantly older than those without lesions. Surprisingly, $H$. pylori infection was present only in $15 / 56$ children $(27 \%)$ and gastrotoxic medications in $13 / 56(23 \%)$. No risk factor was observed in $24 / 56$ children (43\%). The relevance of the study was, however, limited by its short-time duration ( 1 month) and the absence of a control group.

The aim of the present study is to analyze, in a case-control design over a longer period, the risk factors associated with gastric and duodenal ulcers or erosions among pediatric patients referred for upper GI endoscopy in the same European countries. Since age is a major risk factor of gastro-duodenal lesions, to have comparable age distribution, cases and controls were matched for age, center and period.

\section{PATIENTS AND METHODS}

This open, prospective, multicenter, case-control study was carried out during 23 months (January 15, 2008 and December 15, 2009) in 12 centers from 11 European countries. In each center, all the children $<18$ years undergoing an upper GI endoscopy for any indication were enrolled. Data were anonymously reported for patients presenting gastric or duodenal ulcerations and/or erosions and for 2 controls with no ulcer or erosion that immediately followed the index case in the same center, cross-matched for age groups (1-11 months, $1-5$ years, $6-11$ years and $12-17$ years). The following items were recorded: presence of gastric or duodenal 
ulcers and/or erosions, age, gender, known chronic diseases, indications for endoscopy, endoscopic findings, H. pylori status, medications during the last 4 weeks, country of birth of the child and mother, family history of ulcer, lifestyle habits including smoking, alcohol and narcotic consumptions. Children $<1$ month and $>17$ years were excluded. Parental informed consent and child assent were obtained as requested by local ethical policy in the different European countries for the collection of clinical information in an anonymous form. The ethical committee of Queen Fabiola Children's University Hospital, Brussels, Belgium approved the protocol for anonymous data collection and analysis.

$H$. pylori status was determined according to various diagnostic methods as already defined: histology according to the updated Sydney classification, culture, rapid urease test, ${ }^{13} \mathrm{C}$-urea breath test and monoclonal stool antigen. $H$. pylori infection was confirmed when culture or at least 2 other diagnostic tests were positive. ${ }^{21}$

To limit interindividual interpretation of endoscopic findings between observers in the different centers, a clear description and definition of ulcers and erosions was adopted by consensus according to the minimal standard terminology for digestive endoscopy: ulcer was defined as a deep defect in the mucosa with an inflamed edge; erosion was defined as a small $(<3 \mathrm{~mm})$ superficial defect in the mucosa of a white or yellow. ${ }^{22}$

\section{Data Analysis}

The statistical analysis was done using Statistical Package for Social Sciences, version 21.0 (SPSS, IBM, Armonk, NY). The medians and the ranges were calculated for all continuous variables as well as repartition for qualitative parameters. Children having received antimicrobials, proton pump inhibitors or $\mathrm{H}_{2}$-blockers during the preceding 4 weeks were excluded for the analysis of risk. The distributions of nongaussian continuous variables between 2 samples were compared using the nonparametric Mann-Whitney $U$ test. For qualitative variables, monovariate and multivariate statistical analyses were done by logistic regression with adjustment for centers and age groups. The presence of gastric and/or duodenal ulcers or erosions (cases) and the different types of lesions (gastric ulcer, duodenal ulcer, gastric and duodenal ulcer, gastric erosions, duodenal erosions, gastric and duodenal erosions) were introduced successively as dependent variables. The covariates were $H$. pylori infection, gender, known associated chronic diseases, use of medication, lifestyle and familial factors as described in our previous article. ${ }^{1} \mathrm{~A}$ $\chi^{2}$ for homogeneity between centers was also calculated for each significant covariate. A calculation of the size of the population needed to test the hypothesis that gastro-duodenal lesions are mainly caused by H. pylori was first done using the data of our pilot study and a crude estimation of a $H$. pylori frequency of $15 \%$ in controls. Using these settings, 81 patients and 162 controls were needed for ulcers and the same numbers for erosions to obtain a statistically significant difference. Results were expressed as odds ratio (OR) with their $95 \%$ confidence intervals $(95 \% \mathrm{CI})$. Statistical significance was set up at the $P<0.05$ level, and all $P$ values were 2 tailed.

\section{RESULTS}

A total of 732 children ( 244 cases and 488 controls crossmatched for age groups) were enrolled, 383 boys and 349 girls, with a median age of 11.1 years, range 1 month to 17.8 years and interquartile range extending from 6.8 to 14.4 years. Of these 244 enrolled children with gastric and/or duodenal lesions, 102 were included by the Belgian center (and 204 controls), 48 by the Polish center (and 96 controls), 23 by the Swedish center (and 46 controls), between 10 and 20 each by the German and the French centers (and 2 age-matched controls from the same center for each case) and $<10$ each by the Czech, Greek, Hungarian, Italian, Spanish and
Dutch centers (and 2 age-matched controls from the same center for each case). The main indications for upper GI endoscopy were ( $>1$ possible): abdominal pain (113/244 cases vs. $196 / 488$ controls, nonsignificant [NS]), epigastric tenderness (82/244 vs. 148/488, NS), failure to thrive (45/244 vs. $112 / 488$, NS), vomiting $(52 / 244$ vs. $79 / 488$, NS), heartburn ( $28 / 244$ vs. $82 / 488$, NS), chronic diarrhea $(23 / 244$ vs. $41 / 488, N S)$, halitosis $(23 / 244$ vs. $41 / 488, N S)$ and nausea (18/244 vs. 44/488, NS).

The H. pylori status was determined in $708 / 732$ children: by means of gastric biopsies in 707/732 children (232/244 cases and 475/488 controls) and by means of 2 concordant noninvasive tests in 1 control child. In the remaining 24 children, $H$. pylori status was not determined since biopsies could not be obtained due to several contraindications (such as emergency endoscopies for foreign body retrieval or hemorrhage, anticoagulant therapy, hemodynamically compromised children in intensive care unit) and noninvasive tests were not performed. Histologic studies were performed in the biopsy samples of all children, whereas culture was obtained in 581/707 (82.2\%), rapid urease test in $346 / 707$ $(48.9 \%),{ }^{13} \mathrm{C}$-urea breath test in $67 / 707(9.5 \%)$ and stool antigen in 49/707 (6.9\%). Children having received either antimicrobials (21 cases and 29 controls), proton pump inhibitors (16 cases and 52 controls) or $\mathrm{H}_{2}$-blockers ( 5 cases, 17 controls) during the preceding 4 weeks were excluded for the analysis because these medications could have masked the severity of the lesions and could have interfered with $H$. pylori status determination. The characteristics of the remaining 592 children (202 cases and 390 controls) are shown in Table 1. As shown, in a monovariate model, exposure factors associated with an increasing risk of lesions were male gender, $H$. pylori infection, chronic renal diseases, NSAIDs use and active smoking. Conversely, absence of concomitant medication and celiac disease were significantly more frequent in controls. In a multivariate analysis, significant independent factors associated with gastro-duodenal lesions were $H$. pylori infection (OR: $1.911,95 \%$ CI: $1.221-$ 2.992, $P=0.005$ ), male gender (OR: $1.788,95 \%$ CI: $1.220-2.620$, $P=0.003$ ), chronic renal diseases (OR: $3.842,95 \%$ CI: 1.148 $12.854, P=0.029)$ and active smoking (OR: $4.936,95 \% \mathrm{CI}: 1.418$ $17.180, P=0.012)$. Gastro-duodenal ulcers/erosions are more frequent in the older age groups and there is a positive correlation between age and H. pylori infection as shown in Figure 1.

\section{Gastric and Duodenal Ulcers}

Ulcers were observed in 71/202 children (gastric ulcers in 34 , duodenal ulcers in 34 and both gastric and duodenal ulcers in 3 ). Exposure factors associated with gastric ulcers or duodenal ulcers in a monovariate model are shown in the Table 2 and in a multivariate model in Table 3. Significant independent factors associated with gastric ulcers were male gender, chronic neurologic disease, chronic renal disease and NSAID use. Independent factors associated with duodenal ulcers were $H$. pylori infection and steroid use. No independent exposure factors could be associated with ulcerations in both the gastric and duodenal mucosa.

\section{Gastric and Duodenal Erosions}

Erosions alone were observed in 131/202 children (gastric erosions in 83 , duodenal erosions in 38 , gastric and duodenal erosions in 10). Exposure factors associated with these lesions in a monovariate model are shown in the Table 2 and in a multivariate model in Table 3 . The only significant independent factor associated with gastric erosions was chronic renal disease. Independent factors associated with duodenal erosions were $H$. pylori infection, active smoking and chronic arthritis. Independent exposure factors associated with gastric and duodenal erosions were active smoking and a previous successful $H$. pylori eradication. 
TABLE 1. Monovariate Analysis of Exposure Factors in Children With Gastro-duodenal Ulcer and/or Erosions Compared to Controls After Exclusion of Patients Having Received Antimicrobials, PPIs or $\mathrm{H}_{2}$-blockers During the Last 4 Weeks

\begin{tabular}{|c|c|c|c|c|c|c|}
\hline & Cases $(\mathrm{n}=202)$ & Controls $(\mathrm{n}=390)$ & OR & Adjusted* OR & 95\% CI adjusted* OR & $P$ \\
\hline Median age (range) in years & $10.9(0.1-17.8)$ & $10.9(0.2-17.8)$ & & & & NS \\
\hline Male & 118 & 190 & 1.479 & 1.489 & $1.049-2.113$ & 0.026 \\
\hline Migrant & $26(12.9 \%)$ & $34(8.7 \%)$ & 1.551 & 1.571 & $0.902-2.734$ & 0.110 \\
\hline Unknown & 1 & 1 & & & & \\
\hline Migrant family & $65(32.3 \%)$ & $117(30.1 \%)$ & 1.111 & 1.073 & $0.715-1.612$ & 0.734 \\
\hline Unknown & 1 & 1 & & & & \\
\hline Previous endoscopy & $45(22.3 \%)$ & $87(22.3 \%)$ & 0.998 & 1.015 & $0.664-1.551$ & 0.946 \\
\hline Previous $H$. pylori eradication & $11(5.8 \%)$ & $8(2.1 \%)$ & 2.842 & 3.125 & $1.194-8.182$ & 0.002 \\
\hline Unknown & 12 & 12 & & & & \\
\hline H. pylori infection & $60(31.6 \%)$ & $72(19.0 \%)$ & 1.962 & 2.006 & 1.329-3.030 & 0.001 \\
\hline Unknown & 12 & 12 & & & & \\
\hline Family history PUD & $59(30.7 \%)$ & $110(28.9 \%)$ & 1.089 & 1.092 & $0.738-1.615$ & 0.660 \\
\hline Unknown & 10 & 10 & & & & \\
\hline Celiac disease & $5(2.5 \%)$ & $36(9.2 \%)$ & 0.250 & 0.226 & $0.083-0.615$ & 0.004 \\
\hline Chronic renal diseases & $8(4.0 \%)$ & $5(1.3 \%)$ & 3.175 & 3.323 & $1.056-10.456$ & 0.040 \\
\hline No associated chronic disease & $107(53.0 \%)$ & $196(50.3 \%)$ & 1.115 & 0.893 & $0.614-1.298$ & 0.552 \\
\hline Allergic diseases (except food allergy) & $12(5.9 \%)$ & $39(10.0 \%)$ & 0.568 & 0.579 & $0.290-1.155$ & 0.121 \\
\hline Inflammatory bowel disease & $18(8.9 \%)$ & $23(5.9 \%)$ & 1.561 & 1.646 & $0.817-3.313$ & 0.163 \\
\hline Food allergy & $16(7.9 \%)$ & $21(5.4 \%)$ & 1.512 & 1.586 & $0.779-3.228$ & 0.204 \\
\hline Chronic neurologic diseases & $10(5.0 \%)$ & $19(4.9 \%)$ & 1.017 & 1.017 & $0.455-2.273$ & 0.968 \\
\hline Chronic pulmonary diseases & $6(3.0 \%$ & $23(5.9 \%)$ & 0.488 & 0.455 & $0.181-1.148$ & 0.095 \\
\hline Chronic liver diseases & $7(3.5)$ & $15(3.8 \%)$ & 0.897 & 0.929 & $0.335-2.576$ & 0.887 \\
\hline Diabetes mellitus & $7(3.5 \%)$ & $9(2.3 \%)$ & 1.520 & 1.579 & $0.565-4.409$ & 0.383 \\
\hline Chronic ENT diseases & $4(2.0 \%)$ & $9(2.3 \%)$ & 0.855 & 0.824 & $0.244-2.779$ & 0.755 \\
\hline Prematurity & $3(1.5 \%)$ & $5(1.3 \%)$ & 1.161 & 1.106 & $0.252-4.852$ & 0.894 \\
\hline Congenital heart disease & $3(1.5 \%)$ & $4(1.0 \%)$ & 1.455 & 1.586 & $0.338-7.448$ & 0.559 \\
\hline GERD & $1(0.5 \%)$ & $6(1.5 \%)$ & 0.318 & 0.286 & $0.033-2.473$ & 0.255 \\
\hline Juvenile polyarthritis & $4(2.0 \%)$ & $3(0.8 \%)$ & 2.606 & 2.951 & $0.629-13.837$ & 0.170 \\
\hline Other chronic diseases $\dagger$ & $19(9.4 \%)$ & $35(9.0 \%)$ & 0.884 & 0.887 & $0.433-1.818$ & 0.744 \\
\hline No drug consumption & $133(65.8 \%)$ & $282(72.3 \%)$ & 0.738 & 0.636 & $0.418-0.968$ & 0.035 \\
\hline NSAIDs & $23(11.4 \%)$ & $24(6.2 \%)$ & 1.959 & 2.054 & $1.100-3.836$ & 0.024 \\
\hline Steroids & $8(4.0 \%)$ & $8(4.0 \%)$ & 1.969 & 1.999 & $0.724-5.520$ & 0.181 \\
\hline Immunosuppressive agents & $4(2.0 \%)$ & $12(3.1 \%)$ & 0.636 & 0.634 & $0.195-2.064$ & 0.449 \\
\hline Occasional alcohol consumption & $16(8.0 \%)$ & $19(4.9 \%)$ & 1.689 & 1.836 & $0.892-3.780$ & 0.099 \\
\hline Unknown & 2 & 2 & & & & \\
\hline Active smoking & $12(6.0 \%)$ & $4(1.0 \%)$ & 6.111 & 6.801 & 2.097-22.057 & 0.001 \\
\hline Unknown & 1 & 1 & & & & \\
\hline
\end{tabular}

Bold indicates statistically significant exposure factors in blood.

* Adjusted for centers and age groups.

$\dagger$ Other chronic diseases (in order of frequency): malignancy, eosinophilic esophagitis, neonatal stress, patients in intensive care unit, organ transplantation, hypothyroidism, down syndrome and other genetic abnormalities, cystic fibrosis, active tuberculosis, metabolic diseases, chronic pancreatitis and sickle cell disease. More than 1 associated disease possible.

PUD, peptic ulcer disease; GERD, gastro-esophageal reflux disease; ENT, ear, nose and throat.

\section{Ulcers and Erosions Occurring in Children not Exposed to an Identified Associated Factor}

In $105 / 202$ children $(52.0 \%)$ presenting with ulcers and/or erosions, the lesions could have been due to 1 of the following factors: H. pylori infection, inflammatory bowel disease, polyarthritis and other rheumatic diseases, chronic renal disease, stress caused by admission in an intensive care unit (including oncology and neonatal units), use of NSAID, steroid, immunosuppressive agent, chronic neurologic disease or tobacco consumption. In 97/202 patients (48.0\%), no exposure factor potentially responsible of gastric and/or duodenal lesions could be identified. Lesions with unexplained etiology were more frequent in the stomach than in the duodenum (68/117-58.1\% vs. $24 / 72-33.3 \%$, respectively, OR: $2.776,95 \%$ CI: $1.505-5.120$, $P=0.002)$. Simultaneous gastric and duodenal mucosal lesions with unexplained etiology were found in 5/13 children (38.5\%).

\section{DISCUSSION}

H. pylori and chronic use of NSAIDs were recognized as the most common causes of gastro-duodenal ulcers in adults. In children, chronic use of NSAIDs is infrequent and mucosal lesions due to H. pylori are thought to need decades to develop after the primary infection. As a consequence, peptic gastro-duodenal lesions are considered as rare in children.

However, in our previous prospective European multicenter study, we found that $8.1 \%$ of dyspeptic children referred for upper GI endoscopy in European centers presented ulcers and/or erosions in the stomach and/or in the duodenum. ${ }^{1-4}$ These lesions were observed mainly around the second decade of life with a high variability among the different centers. In the present study, we analyzed the other exposure factors associated with gastro-duodenal lesions in an age-matched case-control prospective multicenter design. Our results show that $H$. pylori infection is independently associated with duodenal ulcers and duodenal erosions but not with gastric lesions. NSAIDs use is only independently associated with gastric ulcers and only marginally steroids with duodenal ulcers. Additionally, male gender is independently associated with gastric ulcers and active smoking with gastric and with duodenal erosions. Patients with chronic renal diseases have a higher risk of gastric ulcers and gastric erosions, those with chronic neurologic diseases have a higher risk of 

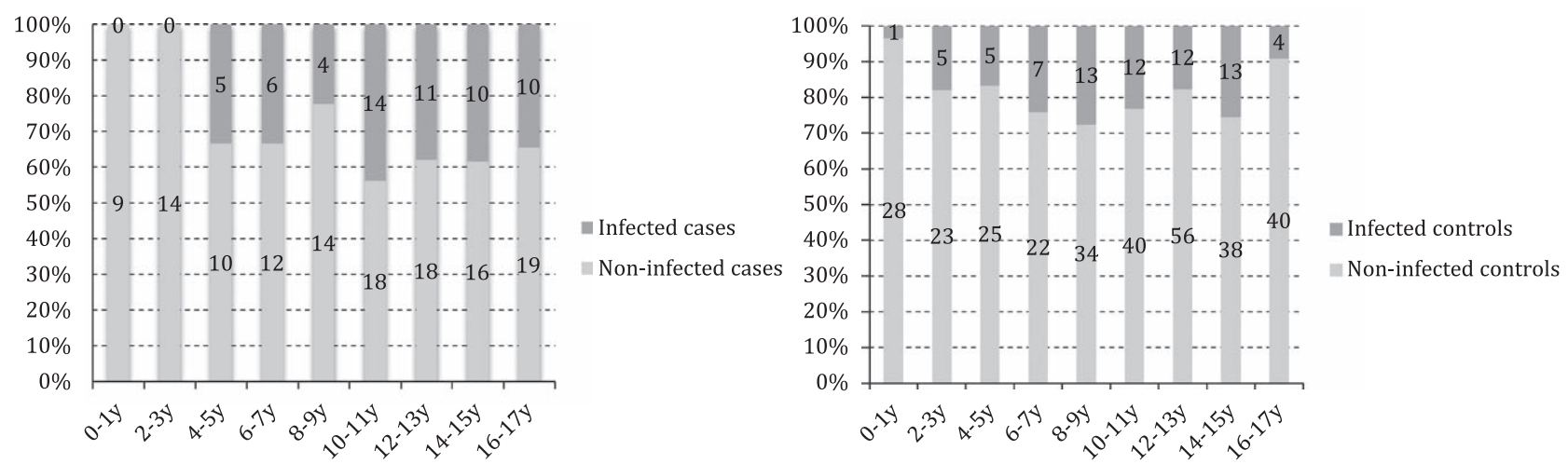

FIGURE 1. Frequency histogram showing the proportion of $H$. pylori infected children, following age groups, among those with gastro-duodenal ulcers/erosions and the controls without lesion. In controls, this proportion remains around $20 \%$, while in children with lesions ("cases") this proportion is very low before 4 years of age, around 30\% between 6 and 10 years and around $40 \%$ in those $>10$ years. Children having received antimicrobials, PPls or $\mathrm{H}_{2}$-blockers during the last 4 weeks were excluded. A significant correlation was found between $\mathrm{H}$. pylori infection and age in children with gastro-duodenal ulcers/ erosions (Spearman's rank correlation $r=0.195, P=0.007)$ but not in controls without lesion $(r=0.013, P=0.795)$.

gastric ulcers, those with chronic juvenile arthritis have a higher risk of duodenal erosions. However, excluding H. pylori, the fractions of cases attributable to these different risk factors were small. Finally, the main finding is the confirmation that ulcers/erosions occur in a large proportion of children that were not exposed to identifiable risk factor in our population and remain currently unexplained. ${ }^{1}$

Potential bias could be the under detection of $H$. pylori infection and the underreported use of medications and/or of etiological factors. However, the diagnostic tests for $H$. pylori were very reliable. The $H$. pylori status was mainly based on concordant histology and culture results (559/581 children, 96.2\%). H. pylori culture was very sensitive in the participating centers with a negative predictive value of $96.6 \%$ : in only 16 children, H. pylori-like microorganisms were identified on histology but culture remained negative while $H$. pylori infection was confirmed by another test. The same applies for histology: in 6 other children, no $H$. pylori-like microorganism was identified histologically although $H$. pylori culture was positive and infection confirmed by another test (negative predictive value of histology for $H$. pylori detection $98.9 \%$ ). Since the $H$. pylori status was not determined in 24 patients (12 cases and 12 controls), we performed the statistical analysis including also the "best case scenario" (we hypothesize they are all infected) and the "worst case scenario" (we hypothesize

TABLE 2. Monovariate Analysis of Exposure Factors in Children With Gastric Ulcer or Duodenal Ulcer or Gastric Erosions or Duodenal Erosions or Gastric and Duodenal Erosions Compared to Controls (Logistic Regression Adjusted for Center and Age Groups) After Exclusion of Patients Having Received Antimicrobials, PPIs or $\mathrm{H}_{2}$-blockers During the Last 4 Weeks

\begin{tabular}{|c|c|c|c|c|c|}
\hline & Gastric Ulcer & $\begin{array}{l}\text { Duodenal } \\
\text { Ulcer }\end{array}$ & Gastric Erosions & $\begin{array}{l}\text { Duodenal } \\
\text { Erosions }\end{array}$ & $\begin{array}{l}\text { Gastric and Duodenal } \\
\text { Erosions }\end{array}$ \\
\hline $\mathrm{N}$ & 34 & 34 & 83 & 38 & 10 \\
\hline Median age (range) in year & $10.1(0.2-17.7)$ & $12.6(1.7-17.1)$ & $10.3(0.1-17.8)$ & $11.7(0.9-17.2)$ & $10.7(2.5-16.8)$ \\
\hline Male & $26(76 \%)^{*}$ & $21(62 \%)$ & $41(49 \%)$ & $21(55 \%)$ & $6(60 \%)$ \\
\hline H. pylori infection & $8(27 \%)$ & $18(53 \%)^{*}$ & $17(22 \%)$ & $13(35 \%) \dagger$ & $4(40 \%)$ \\
\hline Unknown & 4 & 0 & 7 & 1 & 0 \\
\hline No chronic disease & $18(53 \%)$ & $16(47 \%)$ & $48(58 \%)$ & $20(53 \%)$ & $5(50 \%)$ \\
\hline Chronic neurologic diseases & $5(15 \%)^{*}$ & $2(6 \%)$ & $3(4 \%)$ & 0 & 0 \\
\hline Chronic renal diseases & $3(9 \%)^{*}$ & $1(3 \%)$ & $4(5 \%) \dagger$ & 0 & 0 \\
\hline Juvenile polyarthritis & 0 & 0 & $1(1 \%)$ & $3(8 \%)^{*}$ & 0 \\
\hline Other chronic diseases & $3(9 \%)$ & $5(15 \%)$ & 0 & $1(3 \%)$ & $2(20 \%)$ \\
\hline No drug consumption & $22(65 \%)$ & $23(68 \%)$ & $60(72 \%)$ & $24(63 \%)$ & $3(30 \%) \dagger$ \\
\hline NSAIDs & $4(12 \%)$ & $5(15 \%)$ & $8(10 \%)$ & $2(5 \%)$ & $2(20 \%)$ \\
\hline Ibuprofen & 2 & 2 & 3 & 2 & 1 \\
\hline 5-aminosalicylic acids & 0 & 1 & 3 & 0 & 1 \\
\hline Acetylsalicylic acid & 1 & 2 & 0 & 0 & 0 \\
\hline Others & 1 & 0 & 2 & 0 & 0 \\
\hline Steroids & $2(6 \%)$ & $3(9 \%) \dagger$ & $1(1 \%)$ & $1(3 \%)$ & 0 \\
\hline Occasional alcohol consumption & $1(3 \%)$ & $1(3 \%)$ & $7(9 \%)$ & $6(16 \%)^{*}$ & $1(10 \%)$ \\
\hline Unknown & 1 & 0 & 1 & 0 & 0 \\
\hline Active smoking & $1(3 \%)$ & $2(6 \%)$ & $4(5 \%) \dagger$ & $4(11 \%)^{*}$ & $1(10 \%) \dagger$ \\
\hline Unknown & 1 & 0 & 0 & 0 & 0 \\
\hline
\end{tabular}

Bold indicates statistically significant exposure factors.

$* P<0.01$.

$\dagger P<0.05$.

See Table 1 for data of the controls and other chronic diseases. 
TABLE 3. Multivariate Analysis of Exposure Factors in Children With Gastric Ulcer or Duodenal Ulcer or Gastric Erosions or Duodenal Erosions or Gastric and Duodenal Erosions Compared to Controls After Exclusion of Patients Having Received Antimicrobials, PPIs or $\mathrm{H}_{2}-$ blockers During the Last 4 Weeks (Only the Significant Factors Are Shown)

\begin{tabular}{|c|c|c|c|}
\hline & Adjusted* OR & $\begin{array}{c}95 \% \text { CI } \\
\text { Adjusted* OR }\end{array}$ & $P$ \\
\hline \multicolumn{4}{|l|}{ Gastric ulcer } \\
\hline Male & 5.066 & $1.947-13.184$ & 0.001 \\
\hline $\begin{array}{l}\text { Chronic neurologic } \\
\text { disease }\end{array}$ & 4.616 & $1.352-15.758$ & 0.015 \\
\hline Chronic renal disease & 45.799 & $5.357-391.572$ & 0.0005 \\
\hline NSAID & 4.630 & $1.116-19.206$ & 0.035 \\
\hline \multicolumn{4}{|l|}{ Duodenal ulcer } \\
\hline H. pylori infection & 7.535 & $3.119-18.201$ & $<0.001$ \\
\hline Steroids & 6.405 & $1.185-34.625$ & 0.031 \\
\hline \multicolumn{4}{|l|}{ Gastric erosion } \\
\hline Chronic renal disease & 5.496 & $1.223-24.691$ & 0.026 \\
\hline \multicolumn{4}{|l|}{ Duodenal erosion } \\
\hline H. pylori infection & 2.675 & $1.148-6.233$ & 0.023 \\
\hline Active smoking & 14.688 & $2.173-99.286$ & 0.006 \\
\hline Juvenile polyarthritis & 11.587 & $1.888-71.111$ & 0.008 \\
\hline \multicolumn{4}{|c|}{ Gastric and duodenal erosion } \\
\hline $\begin{array}{l}\text { Previous } H \text {. pylori } \\
\text { eradication }\end{array}$ & 22.762 & $2.021-256.369$ & 0.011 \\
\hline Active smoking & 41.540 & $1.506-1145.811$ & 0.028 \\
\hline
\end{tabular}

they are all negative for $H$. pylori) but this did not change the main messages nor the statistical significances.

The study was prospective and a precise questionnaire was used to record the anamnestic data. The questionnaire was carefully reviewed with the children and the families by the investigators. We accept however that intake of some medications could have not been reported: for example tobacco, alcohol and narcotic consumption seems underreported taking into account the current lifestyle of adolescents. Perhaps children may have avoided admitting these consumptions in presence of their parents. However, it is unlikely that such underreported factors could affect half of the children's population. The same could apply for underreported and nondiagnosed chronic diseases such as IBD.

Other monocentric studies recently published show that the incidence of ulcers in children is not negligible, at least in some countries, that these lesions are not only due to H. pylori or gastrotoxic medications and that there is a higher risk for men. In Israel, gastro-duodenal ulcers were detected in 51/751 (6.8\%, $\mathrm{M} / \mathrm{F}=0.9)$ and erosions in $118 / 751(15.7 \%)$ children referred for upper GI endoscopy. The proportion of lesions unrelated to drugs or $H$. pylori infection was only $29 \%$ but in this region the prevalence of $H$. pylori infection is higher than in our population. ${ }^{17}$ In Taiwan, $67 / 1234(5.4 \%, \mathrm{M} / \mathrm{F}=2.5)$ children presented with gastric or duodenal ulcers, $36 \%$ unrelated to drugs or $H$. pylori infection. ${ }^{20}$ In Hong Kong, 51/619 (6.9\%, M/F = 2.6) children presented with gastric or duodenal ulcers, $46 \%$ unrelated to drugs or $H$. pylori infection. ${ }^{18}$ Nevertheless, a much lower frequency (around $2 \%$ ) of ulcers was reported in some other countries..$^{12,14,16}$ The male predominance, found in some studies, may be due to endocrinologic factors..$^{23,24}$ The apparent different prevalence of gastro-duodenal lesions between these different studies may be biased by the fact that the lifestyles are not comparable, unequal availability of endoscopy facilities, referral policy to third level hospitals with endoscopy facilities, total number of endoscopy per year, proportion of older children in each center (lesions more frequent in older ones),
PPI's prescription policy without endoscopy or H. pylori frequency in the local population. Similar bias may explain discrepancies between the different centers participating in the study.

Older studies also described $H$. pylori-negative and NSAIDnegative ulcers/erosions as well as a stronger association of H. pylori infection with duodenal lesions compared with gastric ones. In the first meta-analysis published by Macarthur in 1995, $92 \%$ of children with duodenal ulcer were infected but only $25 \%$ of those with gastric ulcer, while the prevalence of infection in controls with nonulcer dyspepsia was $22 \%$. In more recent pediatric publications and in our study, the prevalence of $H$. pylori in duodenal ulcer is lower. As suggested in adults, it is possible that the declining prevalence of $H$. pylori unmasks patients with peptic ulcer disease unrelated to H. pylori. ${ }^{25,26}$

In contrast to the present study, some authors did not include erosions in their analysis. Since the causes of both lesions are quite similar, we consider that erosions could be an early stage of gastroduodenal damage whose natural evolution can be healing or progression to frank ulcerations.

In previously published articles, treatment with PPIs appears to be effective and recurrence was low even in case of idiopathic lesions but a prospective study must still confirm these data.

We conclude that $H$. pylori infection is an exposure factor associated with duodenal ulcers or erosions, but not with gastric lesions in children. Male gender, NSAIDs, steroids and active smoking or chronic renal diseases, juvenile arthritis as well as chronic neurologic diseases are independent exposure factors associated with gastro-duodenal ulcers/erosions in children. However, a high proportion of children with so-called idiopathic ulcers/erosions have no identified associated risk factor. Further studies are needed to find a causal etiology in these patients such as, perhaps, another exogenous infectious agent or a gastric dysbiosis.

\section{REFERENCES}

1. Kalach N, Bontems P, Koletzko S, et al. Frequency and risk factors of gastric and duodenal ulcers or erosions in children: a prospective 1-month European multicenter study. Eur J Gastroenterol Hepatol. 2010;22:1174-1181.

2. Coghlan JG, Gilligan D, Humphries H, et al. Campylobacter pylori and recurrence of duodenal ulcers-a 12-month follow-up study. Lancet. 1987;2:1109-1111.

3. Ernst PB, Gold BD. The disease spectrum of Helicobacter pylori: the immunopathogenesis of gastroduodenal ulcer and gastric cancer. Annu Rev Microbiol. 2000;54:615-640.

4. Malfertheiner P, Megraud F, O'Morain CA, et al.; European Helicobacter Study Group. Management of Helicobacter pylori infection-the Maastricht IV/florence consensus report. Gut. 2012;61:646-664.

5. Koletzko S, Richy F, Bontems P, et al. Prospective multicentre study on antibiotic resistance of Helicobacter pylori strains obtained from children living in Europe. Gut. 2006;55:1711-1716.

6. Oderda G, Shcherbakov P, Bontems P, et al.; European Pediatric Task Force on Helicobacter pylori. Results from the pediatric European register for treatment of Helicobacter pylori (PERTH). Helicobacter. 2007;12:150-156.

7. Drumm B, Rhoads JM, Stringer DA, et al. Peptic ulcer disease in children: etiology, clinical findings, and clinical course. Pediatrics. 1988;82(3 pt 2):410-414.

8. Oderda G, Vaira D, Holton J, et al. Helicobacter pylori in children with peptic ulcer and their families. Dig Dis Sci. 1991;36:572-576.

9. Macarthur C, Saunders N, Feldman W. Helicobacter pylori, gastroduodenal disease, and recurrent abdominal pain in children. JAMA. 1995;273:729-734.

10. Li Voti G, Acierno C, Tulone V, et al. Relationship between upper gastrointestinal bleeding and non steroidal anti-inflammatory drugs in children. Pediatr Surg Int. 1997;12:264-265.

11. Blecker U, Gold BD. Gastritis and peptic ulcer disease in childhood. Eur J Pediatr. 1999; 158:541-546.

12. Roma E, Kafritsa Y, Panayiotou J, et al. Is peptic ulcer a common cause of upper gastrointestinal symptoms? Eur J Pediatr. 2001;160:497-500.

13. Nijevitch AA, Sataev VU, Vakhitov VA, et al. Childhood peptic ulcer in the Ural area of Russia: clinical status and Helicobacter pylori-associated immune response. J Pediatr Gastroenterol Nutr. 2001;33:558-564. 
14. Elitsur Y, Lawrence Z. Non-Helicobacter pylori related duodenal ulcer disease in children. Helicobacter. 2001;6:239-243.

15. Huang IF, Wu TC, Wang KS, et al. Upper gastrointestinal endoscopy in children with upper gastrointestinal bleeding. J Chin Med Assoc. 2003;66:271-275.

16. Gilger MA, Gold BD. Pediatric endoscopy: new information from the PEDS-CORI project. Curr Gastroenterol Rep. 2005;7:234-239.

17. Egbaria R, Levine A, Tamir A, et al. Peptic ulcers and erosions are common in Israeli children undergoing upper endoscopy. Helicobacter. 2008;13:62-68.

18. Tam YH, Lee $\mathrm{KH}$, To KF, et al. Helicobacter pylori-positive versus Helicobacter pylori-negative idiopathic peptic ulcers in children with their long-term outcomes. J Pediatr Gastroenterol Nutr. 2009;48:299-305.

19. Pacifico L, Anania C, Osborn JF, et al. Consequences of Helicobacter pylori infection in children. World J Gastroenterol. 2010;16:5181-5194.

20. Huang SC, Sheu BS, Lee SC, et al. Etiology and treatment of childhood peptic ulcer disease in Taiwan: a single center 9-year experience. J Formos Med Assoc. 2010;109:75-81.
21. Koletzko S, Jones NL, Goodman KJ, et al; $H$. pylori Working Groups of ESPGHAN and NASPGHAN. Evidence-based guidelines from ESPGHAN and NASPGHAN for Helicobacter pylori infection in children. $J$ Pediatr Gastroenterol Nutr. 2011;53:230-243.

22. Delvaux M, Crespi M, Armengol-Miro JR, et al. Minimal standard terminology for digestive endoscopy: results of prospective testing and validation in the GASTER project. Endoscopy. 2000;32:345-355.

23. Houben CH, Chiu PW, Lau JY, et al. Duodenal ulcers dominate acute upper gastrointestinal tract bleeding in childhood: a 10-year experience from Hong Kong. J Dig Dis. 2008;9:199-203.

24. Smith A, Contreras C, Ko KH, et al. Gender-specific protection of estrogen against gastric acid-induced duodenal injury: stimulation of duodenal mucosal bicarbonate secretion. Endocrinology. 2008;149:4554-4566.

25. Arents NL, Thijs JC, van Zwet AA, et al. Does the declining prevalence of Helicobacter pylori unmask patients with idiopathic peptic ulcer disease? Trends over an 8 year period. Eur J Gastroenterol Hepatol. 2004;16:779-783.

26. Groenen MJ, Kuipers EJ, Hansen BE, et al. Incidence of duodenal ulcers and gastric ulcers in a Western population: back to where it started. Can J Gastroenterol. 2009;23:604-608. 